\title{
A 3D numerical study of the collateral capacity of the circle of Willis with anatomical variation in the posterior circulation
}

\author{
Yuan Ren ${ }^{1}$, Qiang Chen ${ }^{1}$, Zhi-Yong Li ${ }^{1,2^{*}}$
}

\footnotetext{
* Correspondence: zylicam@gmail. com

${ }^{1}$ Biomechanics Laboratory, School of Biological Science \& Medical Engineering, Southeast University, Nanjing 210096, China
}

\begin{abstract}
Background: The Circle of Willis (CoW) is the most important collateral pathway of the cerebral artery. The present study aims to investigate the collateral capacity of CoW with anatomical variation when unilateral internalcarotid artery (ICA) is occluded.

Methods: Basing on MRI data, we have reconstructed eight 3D models with variations in the posterior circulation of the CoW and set four different degrees of stenosis in the right ICA, namely $24 \%, 43 \%, 64 \%$ and $79 \%$, respectively. Finally, a total of 40 models are performed with computational fluid dynamics simulations. All of the simulations share the same boundary condition with static pressure and the volume flow rate (VFR) are obtained to evaluate their collateral capacity.

Results: As for the middle cerebral artery (MCA) and the anterior cerebral artery (ACA), the transitional-type model possesses the best collateral capacity. But for the posterior cerebral artery (PCA), unilateral stenosis of ICA has the weakest influence on the unilateral posterior communicating artery (PCOA) absent model. We also find that the full fetal-type posterior circle of Willis is an utmost dangerous variation which must be paid more attention.

Conclusion: The results demonstrate that different models have different collateral capacities in coping stenosis of unilateral ICA and these differences can be reflected by different outlets. The study could be used as a reference for neurosurgeon in choosing the best treatment strategy.
\end{abstract}

\section{Introduction}

Stroke is a major health problem and a leading cause of adult disability in the world, and it ranks the second in the list of causes of death [1-4]. Wherein, atherosclerosis accounts for up to one-third of strokes [5]. Atherosclerosis of supra-aortic vessels and especially at the common carotid bifurcation is a major cause of recurrent ischaemic stroke [6]. There are two primary reasons for stroke caused by atherosclerosis: one is that the atheromatous plaque may be the source of cerebral emboli, which may result in cerebral infarction [7]. The other is that atherosclerosis leads to vascular stenosis and the decrease of vascular lumen, which may cause inadequate blood perfusion of the downstream cerebrovascular and brain tissue [8].

Carotid occlusive disease amenable to re-vascularization accounts for $5-12 \%$ of new strokes [9-11]. As for patients with severe internal carotid artery (ICA) stenosis, carotid

C 2015 Ren et al: licensee BioMed Central Ltd. This is an Open Access article distributed under the terms of the Creative Commons Attribution License (http://creativecommons.org/licenses/by/4.0), which permits unrestricted use, distribution, and reproduction in any medium, provided the original work is properly cited. The Creative Commons Public Domain Dedication waiver (http:// creativecommons.org/publicdomain/zero/1.0/) applies to the data made available in this article, unless otherwise stated. 
endarterectomy (CEA) has been recognized as an effective therapy for re-vascularization $[12,13]$. However, in fact, it is often compared with carotid artery stenting (CAS), another therapy employed, when selecting the best treatment strategy for an individual patient. Thus, as an important index, the collateral capacity of the cerebral artery is very necessary to be pre-known [14].

The circle of Willis (CoW) is a ring-like arterial structure, and it links the two main cerebral artery systems, namely the internal carotid artery system and the vertebrobasilar system, and it is also the primary collateral pathway locating in the base of brain. When the blood flow of an unilateral artery declines caused by stenosis, it can be compensated from the contralateral side by the CoW. The collateral capacity of the CoW improves cerebral perfusion in ischemic areas and may diminish the effect of ischemic events $[15,16]$. Unfortunately, based on the radiological and anatomical studies $[17,18]$, no more than $50 \%$ of the general population have a complete CoW. The possible variations include hypoplasia or completely absent blood vessels, which may occur in the anterior circulation or the posterior circulation. The variations may influence the collateral capacity of the CoW and the risk of ischaemic stroke. Therefore, evaluating the collateral capacity of different configurable CoWs in patients is very important [19].

There have been some studies performed on haemodynamics of CoW with different anatomical variations in recent years. Some researchers have treated the cerebral vasculature as 1D structure, however, basing on Poiseuille flow, it cannot capture the effects of the complex arterial geometry, especially the effects of blood vessel junctions [20,21]. Although 2D models can improve the accuracy of simulation [22], it's still necessary to establish 3D models for obtaining the more realistic hemodynamic data. There are some existing researches to investigate the collateral capacity of the CoW, based on 3D model $[23,24]$. However, these studies just considered two or three general anatomical variations, in order to understand the influences of other variations, more variational cases should also be paid attention. Moreover, according to the recent CTA study of variations of the CoW, the population of China has a higher prevalence of compromised posterior collateral [25]. Therefore, the present work aims to investigate the collateral capacity of the comprehensive CoW with anatomical variations in the posterior circulation.

\section{Materials and methods}

Basic model reconstruction

Blood of the CoW is supplied by two internal carotid arteries (ICAs) and two vertebral arteries (VAs), and they are classified as the afferent arteries. Each ICA bifurcates to form one middle cerebral artery (MCA) and one anterior cerebral artery (ACA). The two VAs anastomose to form the basilar artery (BA) and then bifurcate to form bilateral posterior cerebral arteries (PCAs). Wherein, bilateral ICAs and PCAs are connected with left and right posterior communicating artery (PCoAs), while bilateral ACAs are connected with anterior communicating artery (ACoA). The ACAs, MCAs and PCAs are classified as the efferent arteries and transport blood away from the CoW to supply the whole cerebral tissue. Figure 1 shows the 3D geometric models of the CoW with the left denoting a patient-specific model of a normal subject and the right representing an ideal model. The patient-specific model was reconstructed with SIMPLEWARE software combined with MRA data. To obtain the spatial feature of the CoW, we extracted the central line from the patient-specific model. With the help of 


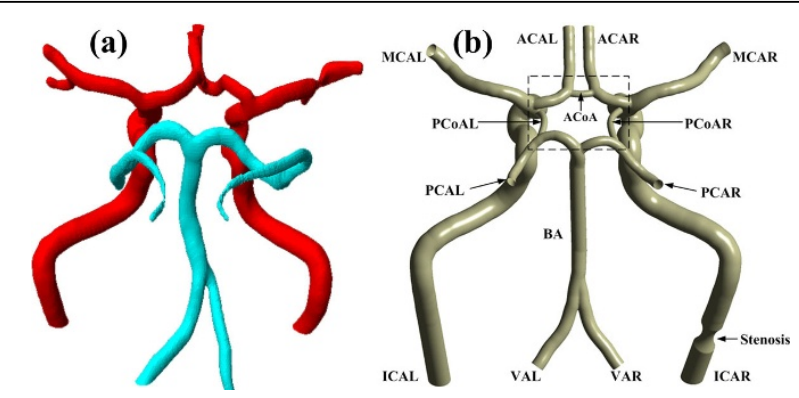

Figure 13 D models of the CoW. (a) Patient-specific model. (b) Ideal model. The abbreviation of each artery branch is marked nearby. The last letter " $L$ " and " $R$ " in the abbreviations represent the left and right side of the arteries, respectively. The black dashed box shows the center of the CoW.

CAD software (ANSYS, Geometry), the ideal CoW model was reconstructed with each vessel branch assigned a constant diameter which was statistized in Ref. [23] basing on a huge amount of MR data.

Based on angiography and anatomical data, variations of the posterior circulation often occurs in the proximal PCA (PCA-P1) and bilateral PCoAs. So we just changed the geometries of these two arteries but kept the remaining arteries constant, including the distal PCA (PCA-P2). The variations of corresponding artery mainly represented the changes of the arterial diameter, and the possible changes might be enlargement, shrinkage or absence. Here, an arterial segment is considered to be normal if it is visible and its diameter is larger than $1 \mathrm{~mm}$, otherwise, it is hypoplastic [18]. Each vessel diameter of the normal model is adopted from Moore et al. [23], and some artificial vessel diameters are introduced to simulate the variable models, which are shown in Table 1. Finally, we obtained eight basic models, including the normal CoW model, as shown in Figure 2, which reflects the anatomical variations in the posterior circulation of the CoW. The detailed definitions of the eight basic models are described below:

(a) Normal type circle (NT): all of the arterial segments are normal, and PCA-P1 has a larger diameter than the PCoA.

(b) Fetal-type posterior (FTP): the diameter of PCA-P1 is smaller than the unilateral PCoA, and the ICA is the main blood supplier to the PCA.

(c) Transitional-type (TransT): the PCoA and PCA-P1 share an equal diameter.

(d) Full FTP (fuFTP): the PCA-P1 is absent, and the PCA arises from the ICA.

(e) Bilateral FTP (bFTP): the variation of FTP occurs on both the two sides.

Table 1 Diameters of each artery branch [23]. Diameter1 and Diameter2 represent the shrunken and enlarged diameters of the corresponding vessels, respectively

\begin{tabular}{cccc}
\hline Vessel & Diameter(mm) & Diameter $\mathbf{( m m})$ & Diameter $\mathbf{( m m})$ \\
\hline ACA & 2.4 & - & - \\
MCA & 2.86 & - & - \\
PCA-P1 & 2.13 & 1.5 & - \\
PCA-P2 & 2.13 & - & - \\
ACoA & 1.47 & - & 2.13 \\
PCoA & 1.45 & 0.7 & - \\
BA & 3.29 & - & - \\
ICA & 4.72 & - & - \\
VA & 2.8 & - & \\
\hline
\end{tabular}




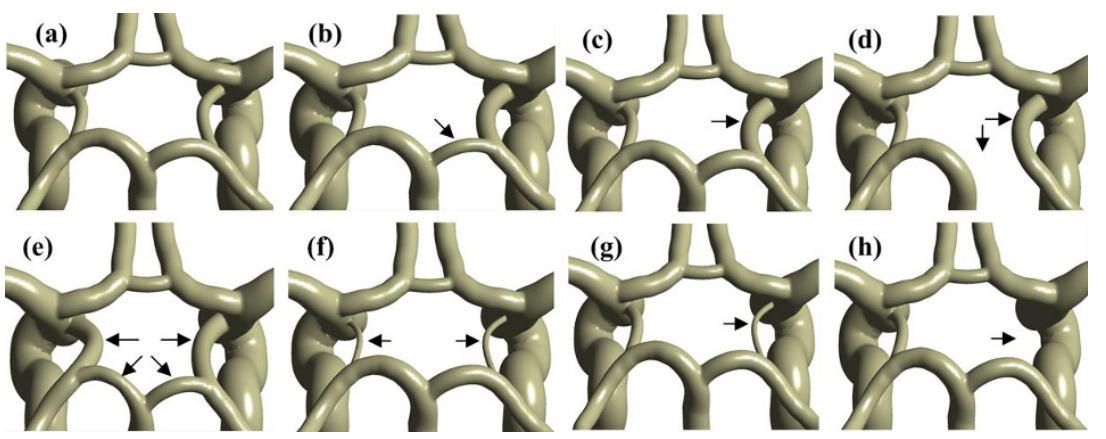

Figure 2 Anatomical variations of the CoW. The black arrows point out the variant arteries compared with the normal CoW model. (a) normal-type circle or NT; (b) fetal-type posterior or FTP; (c) transitionaltype or TransT; (d) full FTP or fuFTP; (e) bilateral FTP or bFTP; (f) bilateral PCoA hypoplasia or bhypPCoA; (g) unilateral PCoA hypoplasia or uhypPCoA; (h) unilateral PCoA absent or uabPCoA.

(f) Bilateral PCoA hypoplasia (bhypPCoA): both the left and right PCoA are hypoplastic, namely, the diameters of bilateral PCoA are less than $1 \mathrm{~mm}$.

(g) Unilateral PCoA hypoplasia (uhypPCoA): only one side of PCoA is hypoplastic, namely, the diameter of unilateral PCoA is less than $1 \mathrm{~mm}$.

(h) Unilateral PCoA absent (uabPCoA): one side of PCoA is absent.

\section{Stenosed model reconstruction}

There are now two major definitions about the stenosis in the ICA. In the North American Symptomatic Carotid Endarterectomy Trial (NASCET), ICA stenosis was classified angiographically: Degree of stenosis $(\mathrm{Std})=(1-$ [narrowest ICA diameter/ diameter normal distal cervical ICA] $\times 100 \%$ [12]. While basing on the same NASCET data, Moneta et al. [26] defined the degree of stenosis in symptomatic patients as the ratio of an internal carotid artery to common carotid artery peak systolic velocity (ICA/CCA PSV). Here, we adopt the first definition of stenosis.

In this work, the stenosed site located in the ICAR, which was marked in Figure 1b, and the stenosed models were obtained by cutting the normal vessel in a revolving way with different semi-elliptical sketches, see Figure 3. Figure 3 depicts the four different severities of stenosis based on the first definition above, namely 24\% (Std24), 43\% (Std43), 64\% (Std64), and 79\%(Std79), respectively. Table 2 lists the geometric parameters of the four sketches. Besides, the maximum cross-area reduction rates
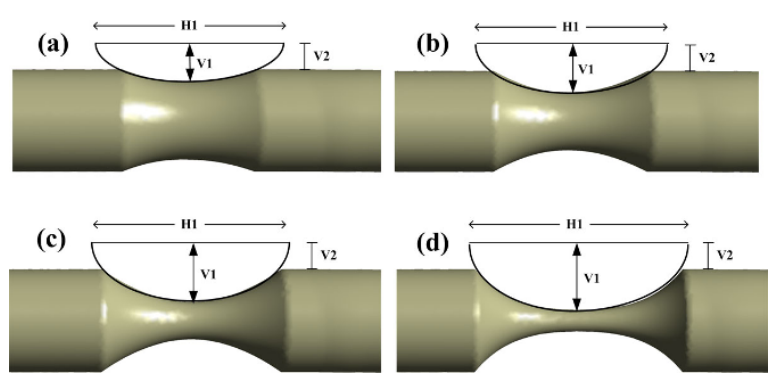

Figure 3 Schematic diagram of the stenosed models: (a) stenosis of $24 \%$ or Std24; (b) stenosis of $43 \%$ or Std43; (c) stenosis of $64 \%$ or Std64; (d) stenosis of 79\% or Std79. Each semi-elliptical sketch is defined with three parameters: $\mathrm{H} 1, \mathrm{~V} 1$ and $\mathrm{V} 2$. 
Table 2 Dimensions of different sketches and the change of MCARR with different stenosis

\begin{tabular}{ccccc}
\hline & $\mathbf{H 1}(\mathbf{m m})$ & $\mathbf{V 1}(\mathbf{m m})$ & $\mathbf{V 2}(\mathbf{m m})$ & MCARR(\%) \\
\hline Std24 & 11 & 1.7 & 1.14 & 42 \\
Std43 & 11 & 2.15 & 1.14 & 67 \\
Std64 & 11 & 2.65 & 1.14 & 87 \\
Std79 & 11 & 3 & 1.14 & 96 \\
\hline
\end{tabular}

(MCARR) are also listed in Table 2. Eventually, each basic model was derived into four stenosed models and totally 40 models or eight groups with different anatomical variations and different severities of stenosis were studied.

\section{CFD Simulation}

Unstructured meshes were created with ICEM CFD (ANSYS, Version 15.0). Element sizes ranged between $0.3 \mathrm{~mm}$ and $0.35 \mathrm{~mm}$, with denser elements in high curvature regions. On average, meshes of each model consisted of 0.47 million nodes and 2.5 million elements. Mesh independence were confirmed by performing a denser mesh than those mentioned above. By comparing the VFR difference between different meshes, the relative errors were less than $3 \%$.

Blood was treated as incompressible, viscous Newtonian fluid, and the vessel wall was assumed to be rigid with no-slip boundary condition. Therefore, the governing equations of blood flow are expressed as:

$$
\frac{\partial \mu}{\partial x}+\frac{\partial v}{\partial y}+\frac{\partial w}{\partial z}=\nabla V=0
$$

and the momentum:

$$
\rho v \cdot \nabla v=-\nabla \cdot \tau-\nabla P
$$

where $P$ is the blood pressure, $v$ is the velocity vector, $\rho$ is the blood density $\left(1056 \mathrm{~kg} / \mathrm{m}^{3}\right)$ and $\tau$ represents the shear stress term. The fluid viscosity is set as 0.004Pa.s.

CFD software Fluent 6.3 (ANSYS) was used to perform the simulations. Blood pressure in the realistic physiological conditions is pulsatile, but it's only change the mean cerebral blood flow [23], so we chose the steady pressure as the inlet and outlet boundary condition. As for the choice of pressure, rather than velocity or mass flow, it's because the change of volume flow rate, which reflects the influence of different models, is the indicator to get. Therefore, six outlets were set as a pressure of 75 $\mathrm{mmHg}$, and the four inlets were set as $82.14 \mathrm{mmHg}$ (ICAs) and $80.17 \mathrm{mmHg}$ (VAs). The reason for the different pressure between ICA and VA is that the pressure drops between the two arteries are different. When we choose the real blood flow measured by PC-MRI as the boundary condition [27], the results verify this difference.

In the simulation, the convergence criterion was satisfied when the residual of continuity was less than $10^{-4}$ and the velocity component is less than 5.0e-5.

\section{Results}

Here, we only considered the influence of the anatomical variations in the posterior circulation and introduced different severities stenosis in the ICAR, so the other conditions were consistently kept. 


\section{Influence of the degree of stenosis}

Figure 4 presents the variation of total flow rate in the four inlets with degree of stenosis from $0 \%$ to $79 \%$ for the eight groups of models. According to a present MRA study based on 125 healthy volunteers, the difference of total flow rate between subjects with normal type circle and PCA-P1 hypoplasia circle (fuFTP in this study) is about $18 \mathrm{ml} /$ $\min (781 \mathrm{ml} / \mathrm{min}$ vs $763 \mathrm{ml} / \mathrm{min})[28]$. Here, in our simulations, we find the corresponding difference is about $20 \mathrm{ml} / \mathrm{min}(590 \mathrm{ml} / \mathrm{min}$ vs $570 \mathrm{ml} / \mathrm{min})$. The difference of total flow rate between our study and the above reference may be that we have neglected some arteries which branch off the ICAs and BAs. In the situation of nostenosis (noStd), the TransT has the maximum flow rate and the fuFTP has the minimum, and the gap between them is only about $24 \mathrm{ml} / \mathrm{min}$. It is worth mentioning that the anatomical variation of CoW may not result in any symptom in clinic. This is because the cerebral arteries have a series of mechanism to maintain the stable blood perfusion, including collateral circulation and cerebral auto-regulation. With the degree of stenosis increases, all of the models have a decline tendency. Especially, it becomes notable when the ICAR is stenosed over $43 \%$. Therefore, the symptoms of cerebral ischemic syndromes may be notable if the degree of unilateral ICA stenosis is greater than $43 \%$.

The blood streamline distributions of the NT model with noStd and Std79 are presented in Figure 5. The Figure 5a, b represent the case with no-stenosis of the ICAR, while the Figure 5c, d represent the case with stenosis of $79 \%$ in the ICAR. In order to visualize the collateral effect when stenosed, we removed the streamline from the stenosed ICAR and presented the rest streamline (Figure 5b, d). From the figures, we can see that the flow rate distribution is approximately symmetrical for the no-stenosis case. There is almost no flow passing through the ACoA, while the weak blood flow through the PCoAs exists from anterior to posterior. However, for the severe stenosis case (79\%), the ipsilateral blood velocity is obviously declined compared with the contralateral blood velocity. In this situation, blood from the ACAL will compensate for the ACAR with the help of the ACoA, and the MCAR will compensated by the PCAR with the help of PCoAR. Meanwhile, we have noticed that there is little blood going through the PCoAL. It can be concluded from the results that the stenosis of ICAR

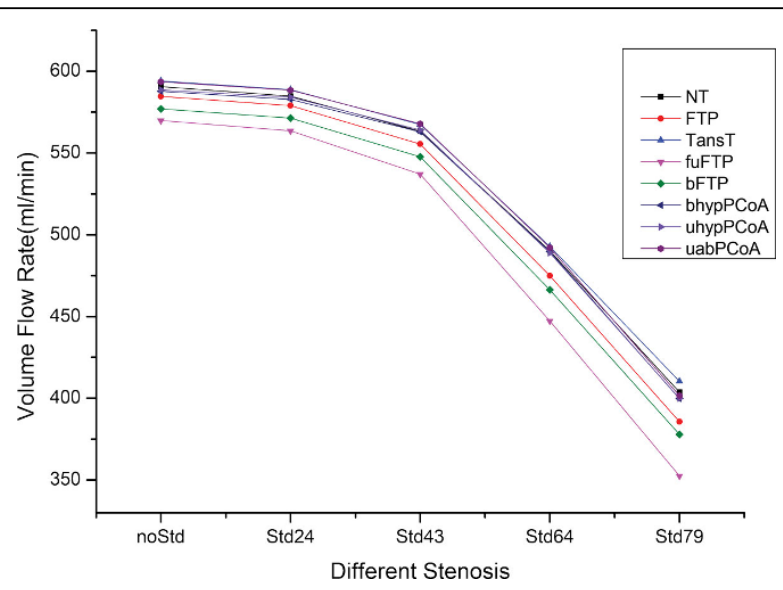

Figure 4 The VFR change of the inlets for the eight models with different degrees of stenosis located in the ICAR. (For the meaning of the different colors, please refer to the online version). 


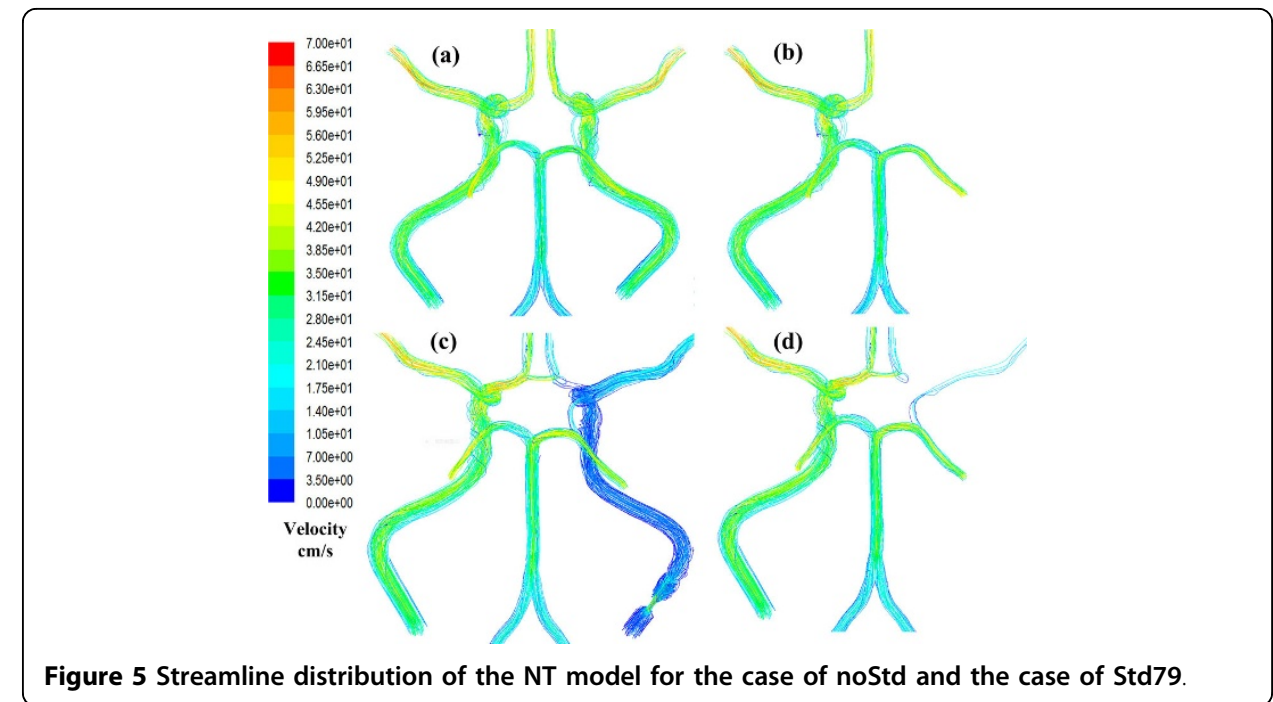

changes the distribution of blood flow and the CoW plays a role in redistributing blood.

\section{Flow rate variations of MCAs\&ACAs with different CoW models}

Because the anatomical variations of the CoW are mainly located in the posterior circulation in this study, namely PCA-P1 and PCoA, the PCAs have different flow variations compared with the ACAs and the MCAs. Moreover, the ACAs and the MCAs are the direct branches of the ICAs, so the stenosis in the ICAR will firstly influence the flow rate of the ACAR and MCAR. Based on the above understanding, we will discuss the PCAs from the ACAs and the MCAs separately.

Figure 6 shows that degree of stenosis results in the reduction of the VFR in the ACA (Black \& Red) and MCA (Blue \& Purple) for the NT model. Regarding the total flow rate, its downward trend is very sharp when the degree of stenosis exceeds $43 \%$ for the ipsilateral ACA and MCA. In contrast, the contralateral ACA and MCA have a weak decline of the VFR, especially for the MCAL. The remaining 7 models share the same tendency with the NT model.

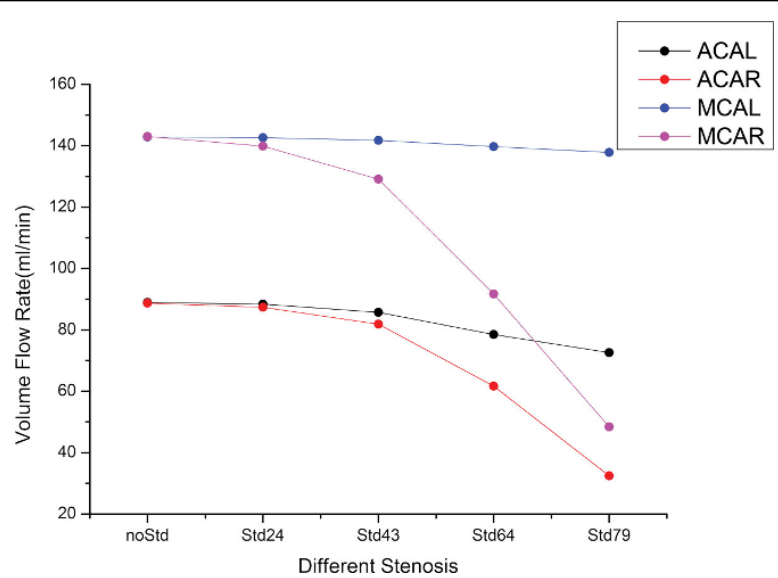

Figure 6 The VFR change of ACAs and MCAs for the NT model with different degrees of stenosis located in the ICAR. 
In order to study the collateral capacity of different models with the severest stenosis (79\%), we defined two parameters: $\xi_{1}$ and $\xi_{2}$. If we take the left anterior cerebral artery (ACAL) and the right anterior cerebral artery (ACAR) as an example, the two parameters can be described as below:

$$
\begin{aligned}
& \xi_{1}=\frac{\text { VFR of ACAL with noStd }- \text { VFR of ACAL with Std79 }}{\text { VFR of ACAL with noStd }} * 100 \% \\
& \xi_{2}=\frac{\text { VFR of ACAR with noStd }- \text { VFR of ACAR with Std79 }}{\text { VFR of ACAL with noStd }- \text { VFR of ACAL with Std79 }}
\end{aligned}
$$

where $\xi_{1}$ denotes the percentage of the blood reduction compared with the no-stenosed model, and $\xi_{2}$ represents the relative collateral capacity of ACAR, compared with ACAL. Basing on this definition, other efferent arteries (MCA\&PCA) can also be studied in similar way. The two parameters of all considered models are compared in Table 3. From the Table 3, we can see that the larger $\xi_{1}$, the more blood reduction, which means a worse collateral capacity; while the relative collateral capacity of MCAR with respect to MCAL is much greater than that of ACAR with respect to ACAL, which means that the ACAR is better than MCAR in the respect of the relative collateral capacity. This is because the connecting artery of ACAR and ACAL (ACoA) is normal, which ensures blood compensation from the ACAL for the ACAR sufficiently. On the contrary, there is no connecting artery between the MCAL and the MCAR, which results in the high sensitivity of the MCAR to the ipsilateral stenosis. If we compare $\xi_{1}$ of the ACAR and MCAR for each model, the difference of blood reduction rate is not noticeable. However, whether it's for ACAR or MCAR, when comparing $\xi_{1}$ for different models, we find that TransT model has the best collateral capacity, followed by the NT model, FTP model and bFTP model, but the collateral capacity of the fuFTP model and uabPCoA model are the worst. As for the parameter $\xi_{2}$, a smaller value means a better relative collateral capacity and a better connection between the left and right side. We can see from Table 3 that the TransT model has the best relative collateral capacity no matter it's for ACA or MCA. However, when it comes to the worst situation for the relative collateral capacity, ACA occurs in the fuFTP model, but MCA occurs in the uabPCoA model.

Flow rate variations of PCAs with different CoW models

Figure 7 presents the changes of VFR of PCAs with different degrees of stenosis for all models. As shown in Figure 7a, flow rate variations of PCAL are not notable with

Table 3 The blood reduction percentage of ACA and MCA compared with their respective no-stenosis model when ICAR stenosed by $79 \%$

\begin{tabular}{ccccccc}
\hline & \multicolumn{3}{c}{$\boldsymbol{\xi}_{\mathbf{1}}$} & & & $\boldsymbol{\xi}_{\mathbf{2}}$ \\
\hline NT & ACAL & ACAR & MCAL & MCAR & ACAR/ACAL & MCAR/MCAL \\
FTP & $18.3 \%$ & $63.5 \%$ & $3.6 \%$ & $66.2 \%$ & 3.45 & 18.55 \\
TansT & $17.0 \%$ & $64.2 \%$ & $3.4 \%$ & $66.3 \%$ & 3.69 & 18.71 \\
fuFTP & $17.1 \%$ & $57.6 \%$ & $3.6 \%$ & $60.5 \%$ & 3.32 & 16.13 \\
bFTP & $16.9 \%$ & $71.3 \%$ & $3.1 \%$ & $72.8 \%$ & 4.07 & 21.20 \\
bhypPCoA & $18.5 \%$ & $63.8 \%$ & $3.2 \%$ & $66.9 \%$ & 3.44 & 21.07 \\
uhypPCoA & $18.9 \%$ & $67.9 \%$ & $3.4 \%$ & $70.3 \%$ & 3.60 & 20.62 \\
uabPCoA & $19.4 \%$ & $67.9 \%$ & $3.5 \%$ & $70.4 \%$ & 3.51 & 3.63 \\
\hline
\end{tabular}



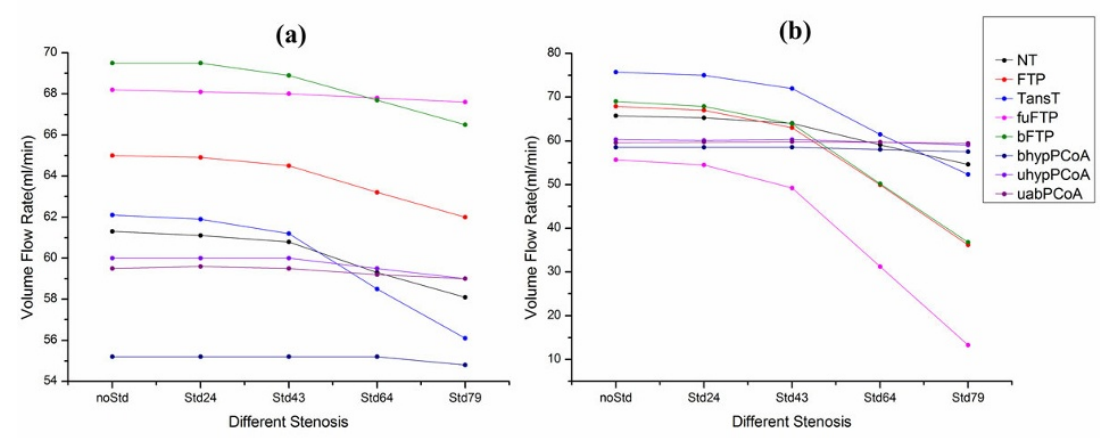

Figure 7 The VFR change of PCAs for the eight models with different degrees of stenosis located in the ICAR. (a) Contralateral PCA (PCAL). (b) Ipsilateral PCA (PCAR). (For the meaning of the different colors, please refer to the online version).

increasing degree of stenosis. For the TransT model, which has the greatest variation, it's only about $6 \mathrm{ml} / \mathrm{min}$ of flow rate reduction in the case of $79 \%$ stenosis. Nevertheless, the flow rate variations of PCARs present a different characteristic, as shown in Figure 7b. Although the degree of stenosis is enlarging, blood flow of PCARs almost remains the same for the uabPCoA, bhypPCoA and uhypPCoA models. In contrast, the fuFTP model presents a feature of a sharp decline.

In order to quantize the flow rate variations of bilateral PCAs, $\xi_{1}$ is shown in table 4 . Comparing the data of different models, we can see that the stenosis (Std79) have the weakest influence on the uabPCoA model, as well as the uhypPCoA and bhypPCoA models. However, it may be fatal to the fuFTP model with $76 \%$ blood reduction rate. Meanwhile, as for the cases of bFTP and FTP models, 79\% stenosis leads to almost half of blood reduction. Comparing the model geometry, anatomical variations of the PCoAR may play an important role in resulting in these different features. If the diameter of PCoAR is hypoplastic or absent, stenosis in the ICAR can hardly influence the ipsilateral PCA, just as the cases of the uabPCoA, uhypPCoA and bhypPCoA models. On the contrary, PCAR of the fuFTP model branches from the ipsilateral ICA, so the influence is directly and considerable.

\section{Discussion and conclusions}

The CoW, served as the primary collateral pathway of the cerebral artery, has a major role in maintaining sufficient blood perfusion for brain tissue. A good collateral

Table 4 The blood reduction percentage of PCAL and PCAR compared with no-stenosis model when ICAR stenosed by $79 \%$

\begin{tabular}{ccc}
\hline & & \\
\hline NT & PCAL & \\
FTP & $5.2 \%$ & PCAR \\
TansT & $4.6 \%$ & $16.9 \%$ \\
fuFTP & $9.7 \%$ & $46.7 \%$ \\
bFTP & $0.9 \%$ & $30.9 \%$ \\
bhypPCoA & $4.3 \%$ & $76.1 \%$ \\
uhypPCoA & $0.7 \%$ & $46.7 \%$ \\
uabPCoA & $1.7 \%$ & $1.7 \%$ \\
& $0.8 \%$ & $2.2 \%$ \\
\hline
\end{tabular}


capacity is beneficial to relieve symptom caused by unilateral artery stenosis or occlusion. The anatomical variations of the CoW lead to the differences for their collateral capacity, and understanding these differences is utmost important for clinicians.

In recent years, the collateral capacity of different configured $\mathrm{CoW}$ has been studied. Long et al. [24] have studied the collateral capacity of the CoW with severe carotid artery based on 3D models, and this literature mainly concerned about the change of pressure. Alastruey et al. [29] have assessed the effects of anatomical variations and occlusions on cerebral flows with 1D computing models, and they have found that ACoA is a more critical collateral pathway than PCoAs if ICA is occluded, and the worst case is that a CoW lacks of the first segment of ACA, and the contralateral ICA is occluded. Fleur et al. [30] have studied the influence of FTP on cerebral collateral circulation, and considered that patients with an FTP may be more prone to suffer vascular insufficiency, especially for the case of full FTP.

In this paper, we have reconstructed eight 3D models with anatomical variations in the posterior circulation, and assessed their collateral capacity in term of the flow rate changes. Compared with other studies, the main contribution of this paper is that the anatomical variations in the posterior circulation were investigated and we have considered more variations. The major concern of this study are blood flow rates of various cerebral arteries, however, there is not enough data based on all of the cases in this study, therefore, we just comparing the distribution of flow of afferent and efferent arteries with published PC-MRI measurements [27,28,31] for some special models, such as the NT and fuFTP models. In the case of NT model with no-stenosis, the VFR of unilateral ICA, MCA and ACA with this measurement are about $210-280 \mathrm{ml} / \mathrm{min}$, $130-155 \mathrm{ml} / \mathrm{min}$ and $75-90 \mathrm{ml} / \mathrm{min}$, respectively. While in our simulation, the corresponding values are $245 \mathrm{ml} / \mathrm{min}, 143 \mathrm{ml} / \mathrm{min}$ and $89 \mathrm{ml} / \mathrm{min}$, respectively. In the case of fuFTP model with no-stenosis, relative contribution by VFR of the ipsilateral ICA, contralateral ICA and BA is 46.6:41.6:11.7, while we find the specific value is 46.5:43:10.5. The present results agree well with the in vivo data in the literature. In fact, because of the auto-regulation of the cerebral arteries, also including the vast collateral anastomoses and individual difference, it is hard to evaluate the collateral capacity of different configured CoWs quantitatively. However, the tendencies reflected by the anatomical variations are helpful in assessing the collateral capacity of the CoW qualitatively.

In conclusion, with the help of CFD simulation, we have found that the collateral capacities of $\mathrm{CoW}$ with anatomical variations in the posterior circulation are different in dealing with unilateral stenosis, and these differences can be reflected by the blood flow rates of efferent arteries. As for the ACAs and MCAs, the TransT model possesses the best collateral capacity. But for the PCAs, unilateral stenosis of ICA has the weakest influence on the uabPCoA model. Meanwhile, we must pay more attention to the fuFTP type for all of the efferent arteries, which have a notable blood flow reduction.

For patients suffering from the ischemic stroke caused by atherosclerosis, it's important to assess the collateral capacity of the cerebral artery, which serves as an important index for selecting the best treatment strategy, especially for the CoW. The study may help neurosurgeons with the risk stratification for patients with cerebral arterial diseases. 
Finally, we have to point out the limitations of the work: (1) the results and conclusions are based on ideal models, not the patient-specific models; (2) pulsatile boundary condition has been ignored.

Competing interests

The authors declare that they have no competing interests.

\section{Authors' contributions}

YR, QC and ZL have made great contributions to the design, analysis and interpretation; YR has been involved in performing the finite element analysis and drafting the manuscript, and QC is responsible for revising it critically; ZL has given final approval of the version and agree to be accountable for all aspects of the work. All authors read and approved the final manuscript.

\section{Authors' information}

YR: M.E., Biomechanics Laboratory, School of Biological Science and Medical Engineering, Southeast University, Nanjing, China; QC: Ph.D, Associate Professor, Biomechanics Laboratory, School of Biological Science and Medical Engineering, Southeast University, Nanjing, China; ZL: Ph.D, Full Professor, Principal Investigator, Biomechanics Laboratory, School of Biological Science and Medical Engineering, Southeast University, Nanjing, China;

\section{Acknowledgements}

This study was partially supported by the National 973 Basic Research Program of China [No.2013CB733800] and the National Natural Science Foundation of China (NSFC) (No. 11272091, 11422222, 31470043, 31300780).

\section{Declarations}

Publication of this article was paid with funding from the National Natural Science Foundation of China (NSFC, No 11272091)

This article has been published as part of BioMedical Engineering OnLine Volume 14 Supplement 1, 2015:

Cardiovascular Disease and Vulnerable Plaque Biomechanics. The full contents of the supplement are available online at http://www.biomedical-engineering-online.com/supplements/14/S1

\section{Authors' details}

'Biomechanics Laboratory, School of Biological Science \& Medical Engineering, Southeast University, Nanjing 210096, China. ${ }^{2}$ School of Chemistry, Physics and Mechanical Engineering, Queensland University of Technology, Brisbane, QLD 4001, Australia.

\section{Published: 9 January 2015}

\section{References}

1. Lloyd-Jones D, Adams R, Carnethon M, et al: Heart disease and stroke statistics-2009 update a report from the American Heart Association Statistics Committee and Stroke Statistics Subcommittee. Circulation 2009, 119(3): e21-e181.

2. Bonita R, Mendis S, Truelsen T, et al: The global stroke initiative. The Lancet Neurology 2004, 3(7):391-393.

3. Durai Pandian J, Padma V, Vijaya P, et al: Stroke and thrombolysis in developing countries. International journal of Stroke 2007, 2(1):17-26.

4. Liu M, Wu B, Wang WZ, et al: Stroke in China: epidemiology, prevention, and management strategies. The Lancet Neurology 2007, 6(5):456-464.

5. Roger VL, Go AS, Lloyd-Jones DM, et al: Heart disease and stroke statistics-2011 update a report from the American Heart Association. Circulation 2011, 123(4):e18-e209.

6. Veith FJ, Amor M, Ohki T, et al: Current status of carotid bifurcation angioplasty and stenting based on a consensus of opinion leaders. Journal of vascular surgery 2001, 33(2):111-116.

7. Amarenco P, Cohen A, Tzourio C, et al: Atherosclerotic disease of the aortic arch and the risk of ischemic stroke. New England Journal of Medicine 1994, 331(22):1474-1479.

8. Goldstein LB, Adams R, Alberts MJ, et al: Primary Prevention of Ischemic Stroke A Guideline From the American Heart Association/American Stroke Association Stroke Council: Cosponsored by the Atherosclerotic Peripheral Vascular Disease Interdisciplinary Working Group; Cardiovascular Nursing Council; Clinical Cardiology Council; Nutrition, Physical Activity, and Metabolism Council; and the Quality of Care and Outcomes Research Interdisciplinary Working Group: The American Academy of Neurology affirms the value of this guideline. Stroke 2006, 37(6):1583-1633.

9. Bogousslavsky J, Van Melle G, Regli F: The Lausanne Stroke Registry: analysis of 1,000 consecutive patients with first stroke. Stroke 1988, 19(9):1083-1092.

10. Inzitari D, Eliasziw M, Gates P, et al: The causes and risk of stroke in patients with asymptomatic internal-carotidartery stenosis. New England Journal of Medicine 2000, 342(23):1693-1701.

11. Timsit SG, Sacco RL, Mohr JP, et al: Early clinical differentiation of cerebral infarction from severe atherosclerotic stenosis and cardioembolism. Stroke 1992, 23(4):486-491.

12. North American Symptomatic Carotid Endarterectomy Trial Collaborators: Beneficial effect of carotid endarterectomy in symptomatic patients with high-grade carotid stenosis. The New England journal of medicine 1991, 325(7):445.

13. Warlow C: MRC European Carotid Surgery Trial: interim results for symptomatic patients with severe (70-99\%) or with mild (0-29\%) carotid stenosis. The Lancet 1991, 337(8752):1235-1243. 
14. Liapis CD, Bell SPRF, Mikhailidis D, et al: ESVS guidelines. Invasive treatment for carotid stenosis: indications, techniques. European journal of vascular and endovascular surgery 2009, 37(4):1-19.

15. Bisschops RHC, Klijn CJM, Kappelle LJ, et al: Collateral flow and ischemic brain lesions in patients with unilateral carotid artery occlusion. Neurology 2003, 60(9):1435-1441.

16. Sallustio F, Kern R, Günther $M$, et al: Assessment of intracranial collateral flow by using dynamic arterial spin labeling MRA and transcranial color-coded duplex ultrasound. Stroke 2008, 39(6):1894-1897.

17. Alpers BJ, Berry RG, Paddison RM: Anatomical studies of the circle of Willis in normal brain. AMA Archives of Neurology and Psychiatry 1959, 81(4):409-418.

18. Kapoor K, Singh B, Dewan LIJ: Variations in the configuration of the circle of Willis. Anatomical science international 2008, 83(2):96-106.

19. Henderson RD, Eliasziw M, Fox AJ, et al: Angiographically defined collateral circulation and risk of stroke in patients with severe carotid artery stenosis. Stroke 2000, 31(1):128-132.

20. Moorhead KT, Doran CV, Chase JG, et al: Lumped parameter and feedback control models of the auto-regulatory response in the circle of Willis. Computer methods in biomechanics and biomedical engineering 2004, 7(3):121-130.

21. Hillen B, Hoogstraten HW, Post L: A mathematical model of the flow in the circle of Willis. Journal of biomechanics 1986, 19(3):187-194.

22. Ferrandez A, David T, Brown MD: Numerical models of auto-regulation and blood flow in the cerebral circulation. Computer Methods in Biomechanics \& Biomedical Engineering 2002, 5(1):7-19.

23. Moore S, David T, Chase JG, et al: 3D models of blood flow in the cerebral vasculature. Journal of biomechanics 2006, 39(8):1454-1463.

24. Long Q, Luppi L, König CS, et al: Study of the collateral capacity of the circle of Willis of patients with severe carotid artery stenosis by 3D computational modeling. Journal of biomechanics 2008, 41(12):2735-2742.

25. Li Q, Li J, Lv F, et al: A multidetector CT angiography study of variations in the circle of Willis in a Chinese population. Journal of Clinical Neuroscience 2011, 18(3):379-383.

26. Moneta GL, Edwards JM, Chitwood RW, et al: Correlation of North American Symptomatic Carotid Endarterectomy Trial (NASCET) angiographic definition of $70 \%$ to $99 \%$ internal carotid artery stenosis with duplex scanning. Journal of vascular surgery 1993, 17(1):152-159.

27. Zhao M, Amin-Hanjani S, Ruland $S$, et al: Regional cerebral blood flow using quantitative MR angiography. American Journal of Neuroradiology 2007, 28(8):1470-1473.

28. Tanaka H, Fujita N, Enoki T, et al: Relationship between variations in the circle of Willis and flow rates in internal carotid and basilar arteries determined by means of magnetic resonance imaging with semiautomated lumen segmentation: reference data from 125 healthy volunteers. American journal of neuroradiology 2006, 27(8):1770-1775.

29. Alastruey J, Parker KH, Peiró J, et al: Modelling the circle of Willis to assess the effects of anatomical variations and occlusions on cerebral flows. Journal of biomechanics 2007, 40(8):1794-1805.

30. van Raamt AF, Mali WPTM, van Laar PJ, et al: The fetal variant of the circle of Willis and its influence on the cerebral collateral circulation. Cerebrovascular Diseases 2006, 22(4):217-224.

31. Rutgers DR, Blankensteijn JD, Van der Grond J: Preoperative MRA flow quantification in CEA patients flow differences between patients who develop cerebral ischemia and patients who do not develop cerebral ischemia during cross-clamping of the carotid artery. Stroke 2000, 31(12):3021-3028.

doi:10.1186/1475-925X-14-S1-S11

Cite this article as: Ren et al:: A 3D numerical study of the collateral capacity of the circle of Willis with anatomical variation in the posterior circulation. BioMedical Engineering OnLine 2015 14(Suppl 1):S11.

\section{Submit your next manuscript to BioMed Central and take full advantage of:}

- Convenient online submission

- Thorough peer review

- No space constraints or color figure charges

- Immediate publication on acceptance

- Inclusion in PubMed, CAS, Scopus and Google Scholar

- Research which is freely available for redistribution 